\title{
Proceeding
}

10th INSHS International Christmas Sport Scientific Conference, 4-5 December 2015. International Network of Sport and Health

Science. Szombathely, Hungary

\section{Correlation between posture and balance in 19-20- year-old albanian students}

\author{
ANDIS BOGDANI ${ }^{1}$, GENTI PANO ${ }^{2}$ \\ ${ }^{1}$ Department of Physical Activity Recreation and Tourism, Sports University of Tirana, Tirana, Albania \\ 2 Department of Physical Activity Health and Recreation, Sports University of Tirana, Tirana, Albania
}

\begin{abstract}
Postural control (or balance) can be defined statically as the ability to maintain a base of support with minimal movement, and dynamically as the ability to perform a task while maintaining a stable position (C. J. Winstein, E. R). The development of equilibrium capacities is known to be critical for both the acquisition and the control of motor skills (Massion. J., 1992). Since balance control is an essential component for the optimal functioning of any locomotors system (Winter DA et al., 1990), gait and balance control together needs to be investigated in order to correct inappropriate performance and prevent injury. This study focus was to investigate the correlation between posture and balance changes in normal and healthy adolescent's (boys and girls). A total of 61 subjects (21 females and 40 males) aged 19-20-year-old students, participated on this study. We have conducted a postural screening, which included digital photography in 4 plans (anterior view, right lateral view, posterior view, left lateral view) and postural evaluation with Posture Screen Mobile program were done, was also performed a set of 4 balance tests BT (Balance Test) a) (Rom EO); b) (Rom EC); c) (SemTanEO); d) (SemTanEC); e) (TanEO); f) (TanEC), on Leonardo Mechanography Platform. Statistical analyses were performed using IBM SPSS Statistics version 2.0. Results shows that generally all subjects have a good balance and orientation level in all tests (with open and closed eyes). Also based on the results we can say that the correlation between posture and balance changes in ages 19-20 it's really relevant. The posture screening and the analysis of balance are of great importance in studies trying to understand the ageing process. Also differences between genders are of great interest especially if a proper exercise program focused in postural improvement can be implemented. Key words: POSTURE, BALANCE, POSTURAL BALANCE.
\end{abstract}

\footnotetext{
Corresponding author. Sports University of Tirana, Sport Science Research Institute, Department of Physical Activity Health and Recreation, Tirana Albania.

E-mail: genti.pano@outlook.com

10th INSHS International Christmas Sport Scientific Conference, 4-5 December 2015. International Network of Sport and Health Science. Szombathely, Hungary.

JOURNAL OF HUMAN SPORT \& EXERCISE ISSN 1988-5202

(c) Faculty of Education. University of Alicante

doi:10.14198/jhse.2016.11.Proc1.16
} 


\section{INTRODUCTION}

Postural control (or balance) can be defined statically as the ability to maintain a base of support with minimal movement, and dynamically as the ability to perform a task while maintaining a stable position (C. J. Winstein, E. R). The development of equilibrium capacities is known to be critical for both the acquisition and the control of motor skills (Massion. J., 1992). Since balance control is an essential component for the optimal functioning of any locomotors system (Winter DA et al., 1990), gait and balance control together needs to be investigated in order to correct inappropriate performance and prevent injury. This study focus was to investigate the correlation between posture and balance changes in normal and healthy adolescent's (boys and girls). Clinically, it is important to measure abnormalities of upright posture. There are several non-invasive methods used to evaluate upright posture, including observation, plumb lines, posture angles on photographs, and orthograms (Beck A, Killus J., 1973; During J et al., 1985; Itoi E., 1991; Raine S, Twomey L., 1994). Therefore, any dichotomous findings between the validity of posture as a cause of pain in published studies might be due to lack of uniform classification and measurement for normal and abnormal posture (Beck $A$, Killus J., 1973; During J et al., 1985; Itoi E., 1991; Raine S, Twomey L., 1994). Since 1970s, segmental spinal movements have been categorized and studied as rotations (Rx, Ry, Rz) and translation (Tx, Ty, Tz) (Panjabi MM et al., 1974). Very few have described posture as head, rib cage, and pelvic rotations and translations (Harrison DD., 1982-1997). Historically, it seems that the postural analysis, as used clinically, is restricted to lateral flexion $(\mathrm{Rz})$, with a minimal evaluation of flexion-extension $(\mathrm{Rx})$ and sagittal balance (Jackson RP, McManus AC., 1994). It has been observed that scoliotic subjects have straightened thoracic curvatures and anterior weight bearing of the thoracic cage (anterior translation=Tz) (Azegami H et al., 1998). Additionally, these subjects often have a low shoulder (thoracic lateral flexion=Rz) and thoracic side shift (lateral translation=Tx) (Azegami H et al., 1998; den Boer WA et al., 1999). We decided to conduct this study because we think that the relationship between posture and balance is of great interest.

\section{Objectives}

This study focus was to investigate the correlation between posture and balance changes in 19-20 years normal and healthy adolescent's (boys and girls).

\section{MATERIAL AND METHODS}

A total of 61 subjects (21 females and 40 males) aged 19-20 year's students at Sports University of Tirana, participated on this study. We have conducted a postural screening, which included digital photography in 4 plans (anterior view, right lateral view, posterior view, left lateral view) and postural evaluation with Posture Screen Mobile program were done, was also performed on Leonardo Mechanography Platform a set of 3 balance tests BT (Balance Test). The subject is trying to stand in a quiet normal standing poition for a certain amount of time (exmpla $10 \mathrm{sec}$ ). For the test to be more difficult are used and we have used 2 other different foot positions, conducted with open eyes and closed eyes. Statistical analyses were performed using IBM SPSS Statistics version 2.0

\section{BT (Balance Test $10 \mathrm{sec}$ ) protocol}

1. Romberg standing position with Eyes Open; 1a Romberg standing position Eyes Closed; (Rom EO; RomEC).

2. Semi Tangent position with Eyes Open; 2a. Semi Tangent position with Eyes closed (SemTanEO); (SemTanEC).

3. Tangent position with Eyes Open; 3a. Tangent position with Eyes Closed (TanEO); (TanEC). 


\section{RESULTS}

\section{Posture results}

Posture Data's reveal the following results that for a total of $(n=61)$ participants the results show: Anterior Translations (1.52 \pm 0.09$)$, Anterior Angulations (4.57 \pm 0.35$)$, Lateral Translations (4.55 \pm 0.23$)$, Lateral Angulations (4.55 .23), Posterior Translations (3.19 \pm 0.17$)$, Posterior Angulations (17.33 \pm 1.86$)$, Lateral Translations $2(4.23 \pm 0.26)$, Lateral Angulations $2(22.92 \pm 1.19)$ variables data sets were summarized and described using descriptive statistics Analyses as presented in table 1.

Table 1: Descriptive Statistics results for Anterior Translations, Anterior Angulations, Lateral Translations 1, Lateral Angulations 1, Posterior Translations, Posterior Angulations, Lateral Translations 2 variables.

\begin{tabular}{lcccccc} 
& N & Minimum & Maximum & \multicolumn{2}{c}{ Mean } & $\begin{array}{c}\text { Std. } \\
\text { Deviation }\end{array}$ \\
& Statistic & Statistic & Statistic & Statistic & $\begin{array}{c}\text { Std. } \\
\text { Error }\end{array}$ & Statistic \\
\hline Anterior Translations & 61 & 0.35 & 3.14 & 1.52 & 0.09 & 0.69 \\
Anterior Angulations & 61 & 0 & 13.2 & 4.57 & 0.35 & 2.7 \\
Lateral Translations Left Side & 61 & 1.47 & 11.95 & 4.55 & 0.23 & 1.82 \\
Lateral Angulations Left Side & 61 & 5.36 & 38.4 & 21.46 & 0.91 & 7.1 \\
Posterior Translations & 61 & 0.13 & 5.97 & 3.19 & 0.17 & 1.31 \\
Posterior Angulations & 61 & 1.5 & 14.8 & 17.33 & 1.86 & 14.53 \\
Lateral Translations Right Side & 61 & 0 & 10.31 & 4.23 & 0.26 & 2.02 \\
Lateral Angulations Right Side & 61 & 0 & 47.8 & 22.92 & 1.19 & 9.26 \\
\hline
\end{tabular}

Descriptive Statistics mean statistic graph is presented in figure 1. Mean values of Lateral Angulations 1, Posterior Angulations and Lateral Angulations 2 reported higher scores compared to the other measured variables.

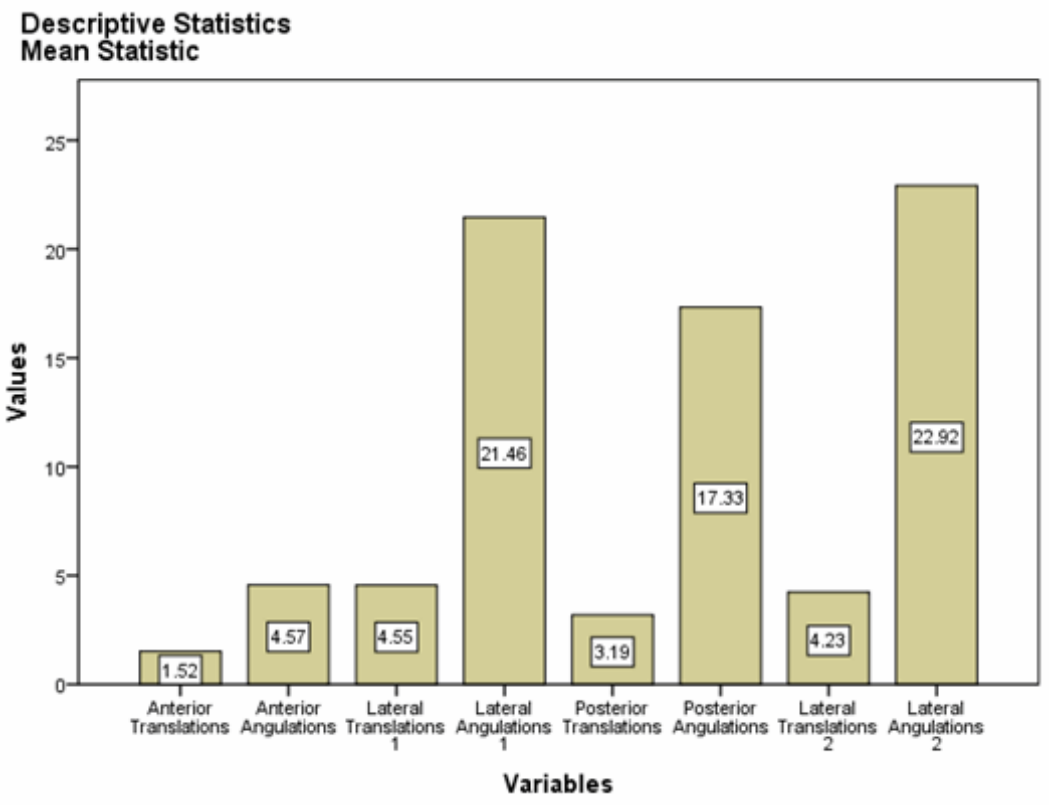

Figure 1. Descriptive Statistics mean statistic graph 
Pearson Correlation. Results revealed a positive significant correlation between: Posterior and Anterior Translations $(n=60, p<0.05)$, Lateral Translations and Lateral Angulations $(n=61, p<0.05)$, Lateral Translations 1 and Lateral Translations $2(n=60, p<0.05)$, Lateral Translations 1 and Lateral Angulations 2 $(n=60, p<0.05)$, Lateral Angulations 1 and Posterior Angulations $(n=60, p<0.05)$, Lateral Angulations and Lateral Angulations $2(n=61, p<0.05)$. Detailed Pearson correlations analyses results are presented in table 2.

Table 2: Pearson Correlations Results for: Anterior Translations, Anterior Angulations, Lateral Translations 1, Lateral Angulations 1, Posterior Translations, Posterior Angulations, Lateral Translations 2 variables.

\begin{tabular}{|c|c|c|c|c|c|c|c|c|}
\hline \multicolumn{9}{|c|}{ Correlations } \\
\hline & & $\begin{array}{c}\text { Anterior } \\
\text { Angulations }\end{array}$ & $\begin{array}{c}\text { Lateral } \\
\text { Translations } \\
1\end{array}$ & $\begin{array}{c}\text { Lateral } \\
\text { Angulations } \\
1\end{array}$ & $\begin{array}{c}\text { Posterior } \\
\text { Translations }\end{array}$ & $\begin{array}{l}\text { Posterior } \\
\text { Angulations }\end{array}$ & $\begin{array}{c}\text { Lateral } \\
\text { Translations } 2\end{array}$ & $\begin{array}{c}\text { Lateral } \\
\text { Angulations } \\
2\end{array}$ \\
\hline \multirow{4}{*}{$\begin{array}{l}\text { Anterior } \\
\text { Translations }\end{array}$} & Pears on Correlation & 0.131 & 0.142 & 0.085 & $.381 * *$ & 0.001 & 0.17 & 0.033 \\
\hline & Sig. (2-tailed) & 0.314 & 0.275 & 0.516 & 0.003 & 0.997 & 0.193 & 0.805 \\
\hline & $\mathrm{N}$ & 61 & 61 & 61 & 60 & 60 & 60 & 60 \\
\hline & Pears on Correlation & & 0.178 & 0.183 & 0.211 & -0.07 & 0.115 & 0.106 \\
\hline \multirow{3}{*}{$\begin{array}{l}\text { Anterior } \\
\text { Angulations }\end{array}$} & Sig̣. (2-tailed) & & 0.171 & 0.158 & 0.106 & 0.597 & 0.383 & 0.421 \\
\hline & $\mathrm{N}$ & & 61 & 61 & 60 & 60 & 60 & 60 \\
\hline & Pears on Correlation & & & $.512 * *$ & 0.211 & 0.05 & $.639 \approx *$ & $.428 * \pi$ \\
\hline \multirow{3}{*}{$\begin{array}{l}\text { Lateral } \\
\text { Translations } \\
1\end{array}$} & Sig. (2-tailed) & & & 0 & 0.105 & 0.702 & 0 & 0.001 \\
\hline & $\mathrm{N}$ & & & 61 & 60 & 60 & 60 & 60 \\
\hline & Pears on Correlation & & & & $.348 * *$ & 0.225 & 0.231 & $.288 *$ \\
\hline \multirow{3}{*}{$\begin{array}{l}\text { Lateral } \\
\text { Angulations } \\
1\end{array}$} & Sig. (2-tailed) & & & & 0.006 & 0.085 & 0.075 & 0.026 \\
\hline & $\mathrm{N}$ & & & & 60 & 60 & 60 & 60 \\
\hline & Pears on Correlation & & & & & 0.101 & 0.168 & 0.08 \\
\hline \multirow{3}{*}{$\begin{array}{l}\text { Posterior } \\
\text { Translations }\end{array}$} & Sig. (2-tailed) & & & & & 0.441 & 0.196 & 0.541 \\
\hline & $\mathrm{N}$ & & & & & 61 & 61 & 61 \\
\hline & Pears on Correlation & & & & & & 0.021 & 0.195 \\
\hline \multirow{3}{*}{$\begin{array}{l}\text { Posterior } \\
\text { Angulations }\end{array}$} & Sig̣. (2-tailed) & & & & & & 0.875 & 0.132 \\
\hline & $\mathrm{N}$ & & & & & & 61 & 61 \\
\hline & Pears on Correlation & & & & & & & .813 * \\
\hline \multirow{2}{*}{$\begin{array}{l}\text { Lateral } \\
\text { Translations } \\
2\end{array}$} & Sig (2-tailed) & & & & & & & 0 \\
\hline & $\mathrm{N}$ & & & & & & & 61 \\
\hline
\end{tabular}




\section{Balance results}

Table 3. Balance Test (BT) RomEO). Analysis Results, Balance Data

\begin{tabular}{|c|c|c|c|c|c|c|c|c|c|c|}
\hline & Age & $\begin{array}{l}\text { Body } \\
\text { Mass }\end{array}$ & $\begin{array}{l}\text { Std. } \\
\text { Elipse } \\
\text { Area } \\
\text { cm }^{2}\end{array}$ & $\begin{array}{c}\text { Std. } \\
\text { Elipse } \\
\text { Angle }\end{array}$ & $\begin{array}{c}\text { num. } \\
\text { Eccentricity }\end{array}$ & $\begin{array}{c}\text { dominat } \\
\text { Freq. } \\
\mathrm{Hz}\end{array}$ & $\begin{array}{c}\text { rel. Path } \\
\text { length } \\
\mathrm{mm} / \mathrm{s}\end{array}$ & $\begin{array}{c}\text { abs. } \\
\text { Path } \\
\text { length } \\
\text { mm }\end{array}$ & $\begin{array}{c}E Q \\
\text { (AP) }\end{array}$ & $\begin{array}{c}\text { Total } \\
\text { Duration } \\
s\end{array}$ \\
\hline Mean & 20.32 & 66.33 & 1.73 & 5.01 & 0.8 & 0.5 & 18.9 & 93.99 & 94.58 & 5 \\
\hline
\end{tabular}

Table 4. Balance Test (BT) RomEC). Analysis Results, Balance Data

\begin{tabular}{|c|c|c|c|c|c|c|c|c|c|c|}
\hline & Age & $\begin{array}{l}\text { Body } \\
\text { Mass }\end{array}$ & $\begin{array}{c}\text { Std. } \\
\text { Elipse } \\
\text { Area } \\
\text { cm }^{2}\end{array}$ & $\begin{array}{c}\text { Std. } \\
\text { Elipse } \\
\text { Angle }\end{array}$ & $\begin{array}{c}\text { num. } \\
\text { Eccentricity }\end{array}$ & $\begin{array}{c}\text { dominat } \\
\text { Freq. } \\
\text { Hz }\end{array}$ & $\begin{array}{c}\text { rel. Path } \\
\text { length } \\
\mathrm{mm} / \mathrm{s}\end{array}$ & $\begin{array}{c}\text { abs. } \\
\text { Path } \\
\text { length } \\
\text { mm }\end{array}$ & $\begin{array}{c}\text { EQ } \\
\text { (AP) }\end{array}$ & $\begin{array}{c}\text { Total } \\
\text { Duration } \\
\mathrm{s}\end{array}$ \\
\hline Mean & 20.32 & 66.33 & 1.75 & 6.78 & 0.82 & 0.53 & 23.3 & 116.51 & 93.32 & 5 \\
\hline
\end{tabular}

Table 5. Balance Test (BT) TanEO). Analysis Results, Balance Data

\begin{tabular}{lcccccccccc} 
& Age & $\begin{array}{c}\text { Body } \\
\text { Mass }\end{array}$ & $\begin{array}{c}\text { Elipse } \\
\text { Area } \\
\text { cm }^{2}\end{array}$ & $\begin{array}{c}\text { Std. } \\
\text { Elipse } \\
\text { Angle }\end{array}$ & $\begin{array}{c}\text { num. } \\
\text { Eccentricity }\end{array}$ & $\begin{array}{c}\text { dominat } \\
\text { Freq. } \\
\text { Hz }\end{array}$ & $\begin{array}{c}\text { rel. Path } \\
\text { length } \\
\mathbf{m m} / \mathbf{s}\end{array}$ & $\begin{array}{c}\text { abs. } \\
\text { Path } \\
\text { length } \\
\text { mm }\end{array}$ & $\begin{array}{c}\text { EQ } \\
\text { (AP) }\end{array}$ & $\begin{array}{c}\text { Total } \\
\text { Duration } \\
\text { s }\end{array}$ \\
\hline Mean & 20.32 & 66.33 & 3.24 & 2.19 & 0.84 & 0.69 & 48.49 & 242.49 & 87.45 & 5 \\
\hline
\end{tabular}

Table 6. Balance Test (BT) TanEC). Analysis Results, Balance Data

\begin{tabular}{|c|c|c|c|c|c|c|c|c|c|c|}
\hline & Age & $\begin{array}{l}\text { Body } \\
\text { Mass }\end{array}$ & $\begin{array}{l}\text { Std. } \\
\text { Elipse } \\
\text { Area } \\
\text { cm }^{2}\end{array}$ & $\begin{array}{c}\text { Std. } \\
\text { Elipse } \\
\text { Angle }\end{array}$ & $\begin{array}{c}\text { num. } \\
\text { Eccentricity }\end{array}$ & $\begin{array}{c}\text { dominat } \\
\text { Freq. } \\
\text { Hz }\end{array}$ & $\begin{array}{c}\text { rel. Path } \\
\text { length } \\
\mathrm{mm} / \mathrm{s}\end{array}$ & $\begin{array}{c}\text { abs. } \\
\text { Path } \\
\text { length } \\
\text { mm }\end{array}$ & $\begin{array}{c}\text { EQ } \\
\text { (AP) }\end{array}$ & $\begin{array}{c}\text { Total } \\
\text { Duration } \\
\mathrm{s}\end{array}$ \\
\hline Mean & 20.32 & 66.33 & 14.35 & 3.80 & 2.02 & 0.42 & 86.70 & 848.60 & 82.1 & 10 \\
\hline
\end{tabular}

\section{DISCUSSION}

Postural analysis with the Posture Screen Mobile is a computerized method, and for this reason we might have small error calculations. Results shows that generally all subjects have a good balance and orientation level in all tests (with open and closed eyes). Also Dunk et al., 2004 performed a reliability study of a photographic technique and consequent digitization of reflective landmarks with 14 participants and reported poor to moderate ICCs for posture reliability. In another reliability study of posture, using an ultrasonic digitizer (Zebris) method of cervical range of motion measurements, Strimpakos et al., 2005 stated that their method employed for measuring cervical joint position sense was unreliable. It has been observed that all the subjects did not have any major postural problems. Subjects in general have normal thoracic curvatures and anterior weight bearing of the thoracic cage. Also based on the results we can say that the correlation between posture and balance changes in ages 19-20 it's really relevant.

\section{CONCLUSIONS}


Based on the posture and balance results we can say that the subjects that have been engaged in physical activity have relatively a good posture with small problems in lateral plane regarding posture and generally a good balance in all the conducted tests. The balance and posture screening analysis are of great importance in studies trying to understand the ageing process. Also differences between genders are of great interest especially if a proper exercise program focused in postural improvement can be implemented.

\section{REFERENCES}

1. Azegami, H., Murachi, S., Kitoh, J., Ishida, Y., Kawakami, N., Makino, M. (1998). Etiology of idiopathic scoliosis. Clin. Orthop. Relat. Res., 357, 229-236.

2. Beck, A., Killus, J. (1973). Normal posture of spine determined by mathematical and statistical methods. Aerospace Med., 44, 1277-1281.

3. den Boer, W.A., Anderson, P.G., Limbeek, J., Kooijman, M.A. (1999). Treatment of idiopathic scoliosis with side-shift therapy: an initial comparison with a brace treatment historical cohort. Eur. Spine J., 8, 406-410.

4. Dunk, N.M., Chung, Y.Y., Compton, Callaghan, J.P. (2004). The reliability of quantifying upright standing postures as a baseline diagnostic clinical tool. J. Manipulative Physiol. Ther., 27, 91-96.

5. During, J., Goudfrooij, H., Keessen, W., Beeker, Th.W., Crowe, A. (1985). Toward standards for posture. Spine, 10, 83-87.

6. Harrison, D.D. (1982-1997) Spinal biomechanics. USA National Library of Medicine \#WE 725 4318C.

7. https://itunes.apple.com/en/app/posturescreen-posture-body/id405109185?mt=8

8. Itoi, E. (1991). Roentgenographic analysis of posture in spinal osteoporotics. Spine, 16, 750-756.

9. Jackson, R.P., McManus, A.C. (1994). Radiographic analysis of sagittal plane alignment and balance in standing volunteers and patients with low back pain matched for age, sex, and size. Spine, 19, 1611-1618.

10. Massion, J. (1992). Movement, posture and equilibrium: Interaction and coordination. Progress in Neurobiology, 38, 35-56.

11. Panjabi, M.M., White, A.A., Brand, R.A. (1974). A note on defining body parts configurations. J. Biomech., 7, 385-390.

12. Raine, S., Twomey, L. (1994). Attributes and qualities of human posture and their relationship to dysfunction or musculoskeletal pain. Crit. Rev. Phys. Rehabil. Med., 6, 409-437.

13. Strimpakos, N., Sakellari, V., Gioftsos, G., Papathanasiou, M., Brountzos, E., Kelekis, D., Kapreli, E., Oldham, J. (2005). Cervical spine ROM measurements: optimizing the testing protocol by using a 3D ultrasound-based motion analysis system. Cephalgia, 25, 1133-45.

14. Strimpakos, N., Sakellari, V., Gioftsos, G., Papathanasiou, M., Brountzos, E., Kelekis, D., Kapreli, E., Oldham, J. (2005). Cervical spine ROM measurements: optimizing the testing protocol by using a 3D ultrasound-based motion analysis system. Cephalgia, 25, 1133-45.

15. Winstein, C.J, Grander, E.R, \& McNeal, D.R. "Standing Balance Training: Effect on Balance: Locomotion in Hemiparetic Adults", Arch. Plays. Med. Rehabil., 70(10), 755-762.

16. Winter, D.A., Patla, A.E., Frank, J.S. (1990). Assessment of balance control in humans. Medical Progress through Technology, 16, 31-51. 